“(C) 2019 IEEE. Personal use of this material is permitted. Permission from IEEE must be obtained for all other uses, in any current or future media, including reprinting/republishing this material for advertising or promotional purposes, creating new collective works, for resale or redistribution to servers or lists, or reuse of any copyrighted component of this work in other works." 


\title{
Recent Breakthroughs on Angle-of-Arrival Estimation for Millimeter-Wave High-Speed Railway Communication
}

\author{
Kai Wu, Wei Ni, Tao Su, Ren Ping Liu and Y. Jay Guo
}

\begin{abstract}
With significantly improved efficiency, large-scale hybrid antenna arrays with tens to hundreds of antennas have great potential to support millimetre-wave (mmWave) communication for high-speed railway (HSR) applications. The significant beamforming gains rely on fast and accurate estimation of the angle-of-arrival (AoA), which however can be impeded by the high train speed, cost/energy oriented design of arrays, and the severe attenuation of $\mathbf{m m W a v e}$ signals. This article reviews these challenges, and discusses the limitations of existing AoA estimation techniques under hybrid antenna array settings. The article further reveals a few recent theoretical breakthroughs which can potentially enable fast and reliable estimation, even based on severely attenuated signals. Under a speed setting of $500 \mathrm{~km} / \mathrm{h}$, a performance study is carried out to confirm the significant improvements of estimation accuracy and subsequent beamforming gains as the results of the breakthroughs.
\end{abstract}

Index Terms-millimeter wave (mmWave); high-speed railway (HSR); phased array; hybrid antenna array; lens antenna array.

\section{Hybrid Antenna ArRay for High-Speed Railway COMMUNICATION}

Being an efficient and sustainable land transport method, future high-speed railway (HSR) is envisaged to be safer, greener and more convenient [1], [2]. Given increased passenger capacity of high-speed trains (HSTs), there are expected to be a substantially increased number of wireless connections between passengers and the Internet. As illustrated in Fig. 1, a practical scenario is to have a train-top antenna array to act as the proxy to relay in-cabin (WiFi, cellular or Ethernet) traffic to track-side base stations (BSs) or communication satellites. Given the scarcity of frequencies and the demand for high data rates of tens of gigabits per second (Gbps) [1], [3], high carrier frequencies with broad bandwidths, such as millimeter-wave (mmWave) or even terahertz $(\mathrm{THz})$, are likely to be used. The mmWave frequency range of $24.25-52.6$ $\mathrm{GHz}$ has been specified in $5 \mathrm{G}$ new radio, and considered in HST scenario [4]. Feasibility studies, including extensive measurement campaigns, have been conducted to confirm the validity of the consideration. For example, a $30 \mathrm{GHz}$ largescale hybrid array has been tested over an HST link between an onboard relay and track-side infrastructure [4]. A significant improvement of spectral efficiency compared with legacy LTEconfigured HST has been demonstrated at the train speed of $500 \mathrm{~km} / \mathrm{h} \mathrm{[4,} \mathrm{Fig.} \mathrm{5].} \mathrm{THz} \mathrm{antenna} \mathrm{arrays} \mathrm{still} \mathrm{have} \mathrm{issues} \mathrm{in}$ compact and efficient design [1], but have started to show a good prospect. For example, a recent work [5] has reported a
$400-\mathrm{GHz} \mathrm{THz}$ antenna array with a measured gain of 33.66 dBi.

\section{A. Large-scale Hybrid Antenna Array}

Large-scale antenna arrays have a range of advantages, e.g., in the mmWave frequency band. The arrays are able to achieve tunable/steerable narrow beams with high gains to combat the severe attenuation the mmWave bands. The narrow beams can also help suppress interference and improve the effective signal-to-interference-plus-noise ratio of intended signals. For example, the beamwidth of a 1,000-element uniform linear array is about $0.0126 \mathrm{rad}$, which is only two percent of the beamwidth of a 20 -element counterpart. The short wavelength of the mmWave frequency makes the integration of hundreds to thousands of antenna elements per array possible [6]. A linear aperture size of $150 \mathrm{~cm}$ can accommodate up to 300 elements with antenna spacing of half wavelength at $30 \mathrm{GHz}$, while it can only accommodate 20 elements at $2 \mathrm{GHz}$.

A hybrid antenna array becomes a preferable and costeffective design of large-scale antenna arrays, where a large number of antennas are grouped and connected into a much smaller number of analog subarrays. Each antenna has an individual configurable phase shifter. Each subarray is connected to a single radio frequency (RF) chain. This is because the physical sizes of RF chains, consisting of analog-to-digital and digital-to-analog converters, power amplifiers, and filters, do not shrink in the mmWave frequency band, as compared to the lower frequencies. Hardware impairments, like different delays in RF components, can be calibrated and suppressed before baseband digital signal processing.

A one-dimensional linear large-scale hybrid array is shown in Fig. 2. The subarrays are typically arranged in a localized fashion to facilitate wiring and schematic design [7]. The subarrays can consist of discrete antenna elements. The resulting array is referred to as Discrete Antenna Array (DAA), which is typically a one-dimensional uniform linear array. In a recent 3GPP HST evaluation [4], a 30-GHz large-scale hybrid array with 256 antennas was installed at an onboard relay. The array is rectangular with four $8 \times 8$ analog subarrays and four $\mathrm{RF}$ chains. The antenna spacing is half of a wavelength. With considerations on energy and cost efficiency, and integration level, a subarray can be replaced with a lens antenna or a Butler matrix, as also shown in Fig. 2.

In the case of lens antenna array, each subarray is a lens antenna array [6], [8]. The lens can focus the microwave 
signals onto its parabola focal surface. By meticulously placing the antennas on the focal surface of the lens, a complete set of discrete Fourier transform (DFT) beams can be activated with sinc-shape beam patterns and pointing directions evenly spaced within $[0,2 \pi)$. Each antenna, referred to as a beam port, can be energized to activate a DFT beam. A switch can be used to select a beam port, and connect the selected beam port to the RF chain hardwired to the switch [6].

In the case of Butler matrix array, each subarray is a Butler matrix which is a passive analog beamforming circuit. The Butler matrix also generates DFT beams. Each beam port can activate an individual DFT beam, like the lens antenna. Also like the lens antenna array, a switch can be used to select and connect a beam port to an RF chain in the Butler matrix arrays. The beam pattern of a DFT beam is a sinc function within $[0,2 \pi)$, as shown on the left-hand side of Fig. 3 . The beam width of the mainlobe depends on the number of antennas. The mainlobe can be shifted (or rotated) by changing the phases of the antennas [8, eq. 7].

The Lens antenna arrays and Butler matrix arrays can be much more energy-efficient than the phase shifter based DAAs due to their high integration level. The lens and Bulter matrix are passive beamformers, while the phase shifters consume non-negligible powers. The typical power consumption of a 4-bit mmWave phase shifter is $30 \mathrm{~mW}$ [6]. To produce a 16-dimensional DFT beam, a total of 16 phase shifters are required, consuming $30 \times 16=480 \mathrm{~mW}$ power. In contrast, the power consumption of an equivalent lens antenna array is only $4 \mathrm{~mW}$ [6].

The one-dimensional Butler matrices and lens antennas can both be readily extended to two-dimensional uniform planar arrays, due to the fact that the DFT beams can be decoupled losslessly between the azimuth and elevation [9]. The DAAs can also be readily extended to two-dimensional uniform planar arrays if DFT beams are adopted. There are also other forms of hybrid antenna arrays, such as fully-connected array and uniform circular array. Overall, they are less tractable due to too many cross-points (in fully connected hybrid arrays) or weak directivity (in uniform circular arrays). Therefore, they are far less popular in practice.

\section{B. Fast Angular Estimation and Tracking of HST}

The train-top array and its counterparts at the track-side BSs or satellites are expected to be large-scale to produce narrow beams with high gains, thereby combating severe attenuation at mmWave frequencies. The arrays are also anticipated to be hybrid with localized architectures in consideration of cost-efficiency and size. Omni-directional transmission could be possible at lower frequencies, but the limited bandwidths available at those frequencies are not wide enough to support Gbps [1]. To this end, accurate estimation and reliable tracking of the angle-of-arrival (AoA) from an HST to the track-side BSs or satellites, or the other way around, is critical to the implementation of the mmWave HSR communication systems.

The AoA estimation is indispensable for efficient utilization of mmWave phased arrays in 5G. It allows for accurately configuring the arrays to quickly capture the impinging signal, form narrow and strong beams, and achieve high signal-tonoise (SNR) and throughput. The fast and accurate AoA estimation can also avoid the round-trip delay of the typical channel estimation and feedback, hence speeding up beamforming and guaranteeing seamless and reliable connectivity.

Conventional channel estimation techniques involving channel sounding, estimation, and feedback would become inadequate. This is because the number of RF chains is much smaller than that of antennas (beams) in mmWave frequency bands given the increasingly compact design of antennas. In contrast, conventional channel estimation approaches were typically designed for digital arrays with the equal numbers of antennas and RF chains in lower frequencies. Important channel information which was accessible to digital arrays, such as the phase difference between antennas, is not readily accessible in the mmWave hybrid antenna arrays. The estimation and feedback could become excessively frequent and likely to be outdated in the HSR application where the trains can travel at very high speeds of up to $500 \mathrm{~km} / \mathrm{h}$. It is important that a train-top mmWave antenna array and its counterpart at the track-side BSs (or satellites) can estimate the AoAs passively and instantly based on the impinging signals from each other, steer and lock their beams, and keep tracking the changes of AoAs to adapt the beams.

\section{Challenge and State of the Art of AoA ESTIMATION FOR LARGE-SCALE HYBRID ARRAYS}

To estimate the AoA in the large-scale hybrid antenna arrays is non-trivial with several key challenges to be properly addressed. None of existing techniques are able to address the challenges holistically. In this section, we start with three key challenges, followed by a review of the state of the art.

\section{A. Estimation Ambiguity}

There is a long-standing estimation ambiguity originating from the architecture of localized hybrid antenna arrays where the received signals at the different antenna elements of a subarray are mixed (or added up) before being sent into an RF chain. The phase difference between adjacent antenna elements, which gives the estimate of the AoA, becomes obscure. Only the phase difference between adjacent subarrays is available, which is a multiple of the inter-element phase difference and needs to be divided by the number of antenna elements in each subarray. Unfortunately, a division of an angle (or phase) gives ambiguous results due to the $2 \pi$ periodicity of angles (or phases).

\section{B. Channel Property}

In most cases, HSTs have unobstructed line-of-sight (LoS) to the track-side BSs. When penetrating buildings and propagating through foliage, mmWave signals attenuate significantly. For these reasons, the mmWave HSR channels are typically dominated by strong LoS paths [1], [3], [10], and can be modeled as Rician channels [11]. Ray-tracing techniques have been employed to simulate the Rician factor - the ratio between the power in the LoS path and the power in the 
scattered paths - in typical outdoor scenarios. As reported in $[10$, Tab. II], the power in the LoS path is at least $11 \mathrm{~dB}$ stronger than the total power in the other paths in the outdoor $30 \mathrm{GHz}$ channels.

The HSR channels can also exhibit strong temporal variations, due to the speed of up to $500 \mathrm{~km} / \mathrm{h}$ of the HSTs [12]. The conventional omnidirectional broadcast is not suitable for the mmWave frequencies, because of severe attenuations at the frequencies. The received SNR can be very low. For example, the received SNR is around $-20 \mathrm{~dB}$ at each antenna, when the carry frequency is $100 \mathrm{GHz}$, the bandwidth is 1 $\mathrm{GHz}$, the transmit power is $20 \mathrm{dBm}$, the transmitter-receiver distance is $100 \mathrm{~m}$, and both the transmit and receive antennas have unit gains [1]. It is necessary to exploit the strong directivity and gains of efficient mmWave antennas to track and uninterruptedly serve the HSTs.

\section{State of The Art}

The two best-known AoA estimation techniques are multiple signal classification (MUSIC) [13] and estimation of signal parameters via rotational invariance techniques (ESPRIT) [13]. Developed originally for full-digital arrays [13], these techniques exploit the orthogonality of the signal and noise subspaces to estimate the signal subspace which is the span of the array response vectors in the directions of the AoAs. MUSIC takes the autocorrelation of received array signals, and estimates the signal subspace as the eigenvectors associated with the large, meaningful eigenvalues of the autocorrelation matrix. ESPRIT divides an array into two subarrays, calculates the transformation matrix between the signal subspaces of the two subarrays, and estimates the AoA from the eigenvalues of the matrix. Computationally expensive singular value decomposition (SVD) is required in both techniques, and could hinder the scalability of the techniques. MUSIC and ESPRIT cannot be directly applied to hybrid antenna arrays, due to the RF combining at analog subarrays and the resultant obscurity of the phase offset information on individual antennas.

Recently, cross-correlation based AoA estimation methods [9], [14], [15] have been developed for hybrid antenna arrays, which extract the AoA information from the cross-correlations of either subarray outputs [9] or the inverse DFT of selected subarray outputs [14], [15]. The methods [9], [14], [15] have low computational complexities, since only a small number of complex multiplications and low-dimensional DFTs are involved. Given these advantages, the cross-correlation based AoA estimation techniques can be potentially suitable for mmWave high-speed railway communications. However, the existing methods [9], [14], [15] suffer from the estimation ambiguities which degrade estimation accuracy, as described in Section II-A.

\section{BREAKTHROUGHS ENABLING FAST TRACKING OF HIGH-SPEED TRAINS}

Despite the estimation ambiguity and long angular search delay, cross-correlation based approaches [9], [14], [15] are still the most appropriate for mmWave HSR communications, due to their strong tolerance to the severe Doppler effect and compatibility with hybrid antenna array structure. New ground-breaking findings are uncovered to eliminate the ambiguity and significantly expedite the estimation and tracking of the AoA within sub-milliseconds.

\section{A. Ground-breaking Discoveries}

One of the significant findings is that, when the analog subarrays point at the angles evenly spaced within $[0,2 \pi)$, there is an underlying deterministic pattern in the crosscorrelations of the received signals between adjacent subarrays at any time [15, Theorem 1]. The pattern can be revealed as follows:

The cross-correlations of received signals between adjacent analog subarrays have consistent signs. The only exception is the strongest cross-correlation which takes the opposite sign from the rest.

The reason behind this finding is that the sign of a crosscorrelation depends on the product of two sine functions which are the denominators of the standard sinc-function beam patterns of the two consecutive subarrays. It has been proved in [15] that the two sine functions take opposite signs, only when the mainlobes of the two consecutive subarrays are on the different sides of the impinging signal. Moreover, the mainlobes of this pair of subarrays are closer to the impinging signal than the mainlobes of the remaining pairs of consecutive subarrays. Therefore, the cross-correlation of the pair is stronger than those of the rest.

This finding is important, as it enables us to quickly and reliably identify the pair of adjacent subarrays providing the cross-correlation with the opposite sign. We can correct the sign, and then coherently combine all the cross-correlations to enhance the SNR for the estimation of the phase difference between adjacent analog subarrays, and estimate the phase difference unambiguously. The finding is also very insightful, as the strongest cross-correlation is the least susceptible to receiver noises and most unlikely to be mistaken.

The finding can be extended to multiple time slots (or training symbols), where angularly evenly spaced DFT beams are produced at every symbol. Only the pairs of consecutive subarrays with their mainlobes on the different sides of the impinging signal have cross-correlations with the opposite sign to the rest of the pairs at each symbol. The use of multiple symbols can collectively narrow down the angular region of the impinging signal.

In the presence of non-negligible noise, estimation inaccuracies can occur. Since the strongest cross-correlation is expected to take the opposite sign at every symbol, they are the least likely to be corrupted by noise. This provides the most reliable and noise-resisting estimation by identifying the pairs of consecutive subarrays which each have the strongest cross-correlation at one of the training symbols and all have an overlapping angular region between their mainlobes. This gives the best possible estimation accuracy of the phase difference between consecutive subarrays.

Another breakthrough is that, when the analog subarrays point at angles evenly spaced within $[0,2 \pi)$, the received signals of the subarrays can be augmented to achieve the unambiguous estimation of the AoA [14], [15, Theorem 2]: 
Provided that the phase offsets between adjacent analog subarrays are perfectly canceled, the received signals of the angularly evenly spaced subarrays form a Fourier series. The phase of the Fourier coefficients provides an unambiguous estimate of the AoA.

The reason behind this finding is that the angularly evenly spaced subarrays receive the same impinging signal using different beam lobes. Since all the subarrays have the same sinc-function beam pattern, the received signals of the subarrays (after the phase differences between the subarrays are removed) can be interpreted as evenly spaced samples of the sinc-function beam pattern, as shown in Fig. 3. This gives a complete Fourier (or DFT) series with an additional phase which can uniquely determine the AoA of the impinging signal.

By following the ground-breaking discoveries, the AoA can be quickly and reliably estimated at a large-scale hybrid antenna array, even in low SNR regimes.

\section{B. Algorithm Implementation}

The two findings can be translated to simple executable operations for the unambiguous estimation of the AoA. The following three steps can be formed:

1) Steer the analog subarrays towards angles evenly spaced angles between $[0,2 \pi)$ during a training symbol (by configuring the phase shifters of the DAAs or selecting the DFT beams of the lens and Butler matrix arrays). If multiple symbols are used, rotate the beams altogether in steps to increase the angular resolution of evenly spaced pointing angles;

2) Take cross-correlations between the received signals of adjacent subarrays,

a) In the case of a single symbol, identify the strongest cross-correlation and invert its sign before additively combining all the cross-correlations;

b) In the case of multiple symbols, utilize the recognizable two-dimensional pattern of the signs of the cross-correlations over the symbols to identify the two cross-correlations with the opposite sign, and invert their signs before additively combining all the crosscorrelations;

and then estimate the phase of the combined crosscorrelations as the phase difference between subarrays;

3) Augment the received signal at every subarray by canceling the inter-subarray phase difference, take the inverse DFT of the augmented signals, and finally estimate the AoA from the phase difference between consecutive Fourier coefficients.

The system framework of the new unambiguous estimation of the AoA is provided in Fig. 4, where Steps 1), 2) and 3) are highlighted in different frames. A single training symbol is taken as an example.

\section{Performance Study}

Numerical studies are provided to evaluate the application of hybrid arrays in mmWave HSR communications. A typical 30$\mathrm{GHz}$ railway system with $500 \mathrm{MHz}$ bandwidth is considered
[10]. A localized hybrid array with 4 subarrays is installed on the roof of the train, where the subarrays can be a phase shifter based DAA, a lens antenna array, or a Butler matrix array. The antennas in the subarrays are omnidirectional with unit gain.

The BS transmits signals to the HST with $30 \mathrm{dBm}$ transmitting power and $25 \mathrm{dBi}$ antenna gain, while the HST travels away from the BS at $500 \mathrm{~km} / \mathrm{h}$ and the distance from the HST and BS grows from $10 \mathrm{~m}$ to $1 \mathrm{~km}$ during this performance study. Based on the RF thermal noise approximation [3], the received SNR of the system at the antenna elements decreases from $20 \mathrm{~dB}$ to $-20 \mathrm{~dB}$, as the transmitter-receiver distance increases from $10 \mathrm{~m}$ to $1 \mathrm{~km}$. We assume a Rician channel with a direct LoS path and two indirect paths. We set the Rician factor to grow with the transmitter-receiver distance. The Rician factor is $10 \mathrm{~dB}$ when the distance is $10 \mathrm{~m}$, and $30 \mathrm{~dB}$ when the distance is $1 \mathrm{~km}$, as suggested by the measurement results [10, Tab. II]. The AoA of the direct LoS path is uniformly distributed in $[0,2 \pi)$ rad. The Doppler frequency follows the Gaussian distribution with the mean of $10 \mathrm{kHz}$ and the standard deviation of $5 \mathrm{kHz}$.

Fig. 5 plots the mean squared error (MSE) of AoA estimates as the received SNR at the hybrid antenna arrays increases, where different number of antennas per subarray are taken. The figure provides the average result of 20,000 independent trials under each SNR value. For each trial, the AoAs of a direct LoS path and two indirect paths, and the Doppler frequency of the direct path are randomly and independently generated. The algorithm described in Section III-B is performed to estimate the AoA of the direct path. We see that the new AoA estimation approach can achieve very low estimation errors, even in low SNR regions. Specifically, the MSE can be as low as $1.6 \times 10^{-4}$ at $-20 \mathrm{~dB}$ SNR, thanks to the new findings unveiled in Section III. We also see that the MSE decreases markedly, as the number of antennas per subarray increases from 8 to 40 . The improvement can be as large as $75.46 \%$. Moreover, as observed in Fig. 4, the AoA estimation accuracy can be improved by increasing the number of antennas in each subarray. Since the number of subarrays is not increased, the computational complexity does not grow with the increasing number of antennas per subarray and the improving estimation accuracy. In other words, the AoA estimation accuracy can be substantially improved by cost-effectively integrating more antennas for each subarray.

Fig. 5 also validates the efficiency and accuracy of the new AoA estimation approach, as compared to the state-of-the-art approach specifically designed for localized hybrid arrays [9]. We see that the new approach can substantially outperform the state of the art. In low SNR regions, the new technique can reduce the MSE of the AoA estimation by as much as $95.99 \%$ with $80 \%$ less number of symbols, as compared to the state of the art. We note that the results apply to all of the DAA, lens antenna array, and Butler matrix array, or any localized hybrid array with subarrays capable of producing DFT beams. Despite their different structures and integration levels, the DAA, lens antenna, and Butler matrix can all produce DFT beams.

Fig. 6 provides the complementary cumulative distribution function (CCDF) of the subarray beamforming gain that is 
calculated based on the AoA estimates at $-20 \mathrm{~dB}$ SNR from Fig. 5. The subarray beaforming gain is obtained by steering all the subarrays to the closet possible angle around the estimated AoA. Despite the phase shifter based DAA, lens and Butler matrix arrays can all achieve the same AoA estimation accuracy, as discussed in Fig. 4. The DAA can accurately form a beam to the estimated AoA; while the lens and Butler matrix can only select the angularly closest DFT beam from their discrete sets of DFT beams, leading to gain losses. Since the lens and Butler matrix arrays are expected to give exactly the same results, they are both represented by the same curve in Fig. 5 to keep the figure clear.

We also see in Fig. 6 that large beamforming gains can be achieved, given the fine AoA estimation accuracy. The beamforming gains based on phase shifters can be greater than $86.58 \%$ of the maximum-achievable gain, i.e., the number of antennas, in no less than $90 \%$ of all cases. We also see that the lens or Butler matrix based beamforming gains can be smaller than the gain based on phase shifters. As mentioned, this is caused by the fixed DFT beamforming of the lens antennas and Butler matrices, which is the price paid for increased system integration level and energy efficiency.

\section{CONCLUSION}

This article reviews existing AoA estimation techniques, and discusses their limitations under mmWave hybrid antenna array and high-speed settings. A few recent theoretical breakthroughs are presented, where the deterministic properties of the complex beamforming gains of hybrid antenna arrays are unveiled. It is demonstrated that the breakthroughs can potentially enable fast and reliable estimation of the AoA, even based on severely attenuated signals in high-speed scenarios. Under a speed setting of $500 \mathrm{~km}$, a performance study shows the significant improvements of estimation accuracy and subsequent beamforming gains as the results of the breakthroughs, even at a low SNR of $-20 \mathrm{~dB}$.

\section{REFERENCES}

[1] K. Guan et al., "On millimeter wave and $\mathrm{THz}$ mobile radio channel for smart rail mobility," IEEE Trans. Veh. Technol., vol. 66, no. 7, pp. 5658-5674, July 2017.

[2] B. Ai et al., "Challenges toward wireless communications for high-speed railway," IEEE Trans. Intell. Transp. Syst., vol. 15, no. 5, pp. 2143-2158, Oct 2014.

[3] K. Guan et al., "Towards realistic high-speed train channels at 5G millimeter-wave band-part II: Case study for paradigm implementation," IEEE Trans. Veh. Technol., vol. 67, no. 10, pp. 9129-9144, Oct 2018.

[4] F. Hasegawa et al., "High-speed train communications standardization in 3GPP 5G NR," IEEE Commun. Standards Mag., vol. 2, no. 1, pp. 44-52, MARCH 2018.

[5] Z. Miao et al., "A 400-GHz high-gain quartz-based single layered folded reflectarray antenna for Terahertz applications," IEEE Trans. Terahertz Sci. Technol., vol. 9, no. 1, pp. 78-88, Jan 2019.

[6] X. Gao et al., "Low RF-complexity technologies to enable millimeterwave MIMO with large antenna array for $5 \mathrm{G}$ wireless communications," IEEE Commun. Mag., vol. 56, no. 4, pp. 211-217, APRIL 2018.

[7] C. Lin and G. Y. L. Li, "Terahertz communications: An array-ofsubarrays solution," IEEE Comm. Mag., vol. 54, no. 12, pp. 124-131, December 2016

[8] K. Wu et al., "Efficient Angle-of-Arrival estimation of lens antenna arrays for wireless information and power transfer," IEEE J. Sel. Areas Commun., vol. 37, no. 1, pp. 116-130, 2019.
[9] X. Huang, Y. J. Guo, and J. D. Bunton, "A hybrid adaptive antenna array," IEEE Trans. Wireless Commun., vol. 9, no. 5, pp. 1770-1779, 2010.

[10] C. Zhang et al., "Doppler shift estimation for millimeter-wave communication systems on high-speed railways," IEEE Access, pp. 1-1, 2018.

[11] I. A. Hemadeh et al., "Millimeter-wave communications: Physical channel models, design considerations, antenna constructions, and linkbudget," IEEE Commun. Surveys Tuts., vol. 20, no. 2, pp. 870-913, Secondquarter 2018.

[12] H. Song et al., "Millimeter-wave network architectures for future highspeed railway communications: Challenges and solutions," IEEE Wireless Commun., vol. 23, no. 6, pp. 114-122, December 2016.

[13] S.-F. Chuang, W.-R. Wu, and Y.-T. Liu, "High-resolution AoA estimation for hybrid antenna arrays," IEEE Trans. Antennas Propag., vol. 63, no. 7, pp. 2955-2968, 2015.

[14] J. A. Zhang et al., "Angle-of-arrival estimation using different phase shifts across subarrays in localized hybrid arrays," IEEE Commun. Lett., vol. 20, no. 11, pp. 2205-2208, Nov 2016.

[15] K. Wu et al., "Robust unambiguous estimation of angle-of-arrival in hybrid array with localized analog subarrays," IEEE Trans. Wireless Commun., vol. 17, no. 5, pp. 2987-3002, May 2018.

Kai Wu received the B.E. in Information Countermeasure Technology and the Ph.D. in Signal and Information Processing from Xidian University, Xi'an, China, in 2012 and 2019, respectively. Currently, he is studying towards a second Ph.D. degree of the University of Technology Sydney (UTS). His research interests include array signal processing, beamforming, and AoA estimation.

Wei Ni (M'09-SM'15) received the B.E. and Ph.D. degrees in Electronic Engineering from Fudan University, Shanghai, China, in 2000 and 2005, respectively. Currently he is a Team Leader at CSIRO, Sydney, Australia, and an adjunct professor at the UTS. Prior to this, he was a postdoctoral research fellow at Shanghai Jiaotong University (2005-2008), Deputy Project Manager at the Bell Labs/Alcatel-Lucent (2005-2008), and Senior Researcher at Nokia (2008-2009).

Tao Su received the B.S. degree in information theory, M.S. degree in mobile communication, and $\mathrm{Ph} . \mathrm{D}$. degree in signal and information processing from Xidian University, Xi' an, China, in 1990, 1993, and 1999, respectively. Since 1993, he has been a Faculty with Xidian University, where he is currently a Professor with the National Laboratory of Radar Signal Processing, School of Electronic Engineering.

Ren Ping Liu (M'09-SM'14) received his B.E. and M.E. degrees from Beijing University of Posts and Telecommunications, China, and the Ph.D. degree from the University of Newcastle, Australia. He is currently a Professor and Head of Discipline of Network \& Cybersecurity at UTS. Professor Liu is also the co-founder and CTO of Ultimo Digital Technologies. Prior to that he was a Principal Scientist and Research Leader at CSIRO.

Y. Jay Guo (Fellow'2014) received a Bachelor Degree and a Master Degree from Xidian University in 1982 and 1984, respectively, and a PhD Degree from Xi'an Jiaotong University in 1987, all in China. Currently, he is a Distinguished Professor and the Director of Global Big Data Technologies Centre at UTS, Australia. Prior to this, he served as a Research Director in CSIRO for over nine years, and held senior leadership positions in Fujitsu, Siemens and NEC in the U.K 


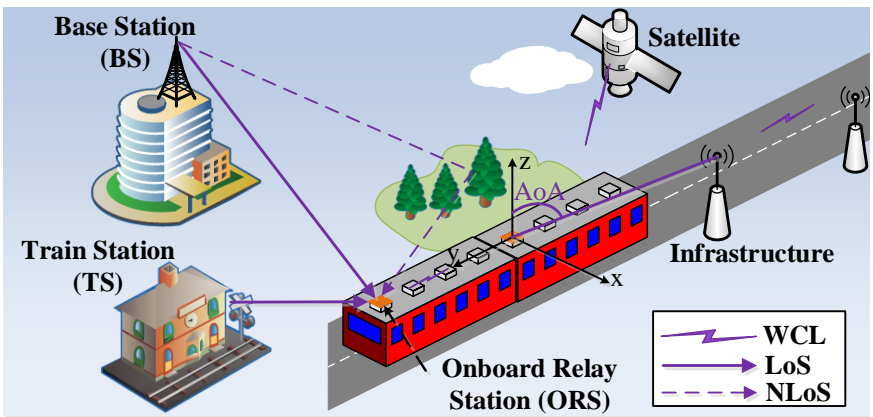

Fig. 1. Schematic diagram of the "smart" railway communication system with the interconnections between the HST and BS, TS, infrastructures, as well as satellite. WCL refers to wireless communication link. 


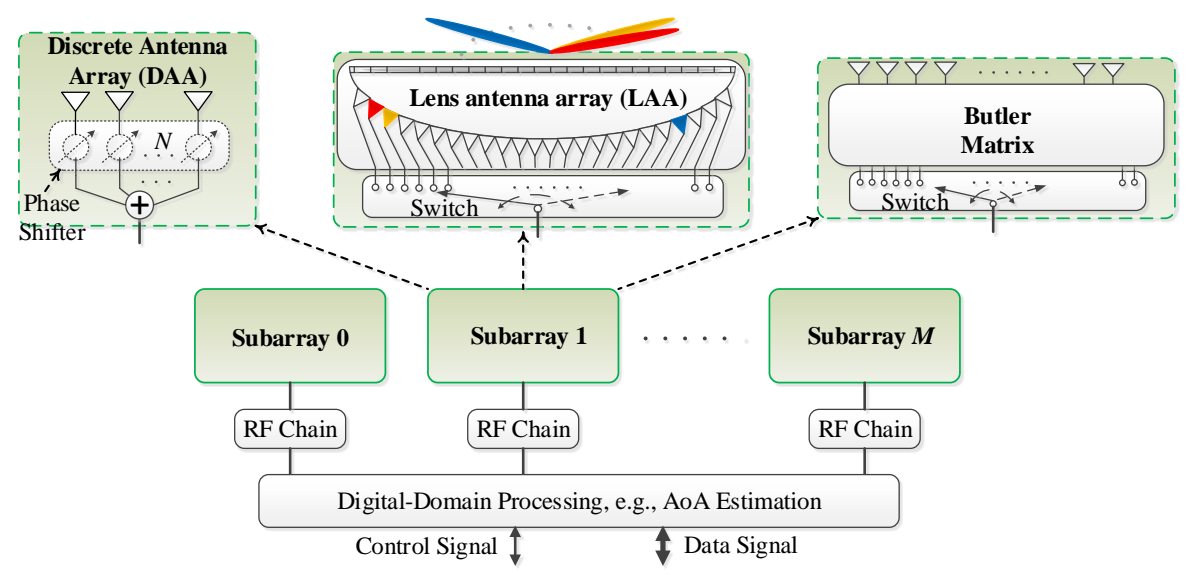

Fig. 2. Schematic diagram of a one-dimensional localized hybrid array, where the subarrays can be implemented by either generic discrete antenna elements, or highly integrated antennas such as lens and Butler matrix. 


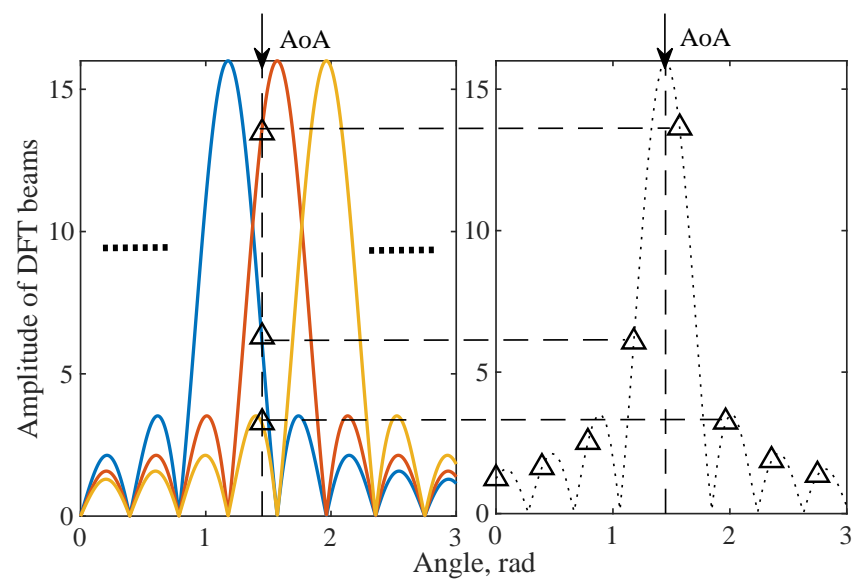

Fig. 3. Illustration of the amplitudes of consecutive DFT beams and the responses of the DFT beams at the target AoA. 


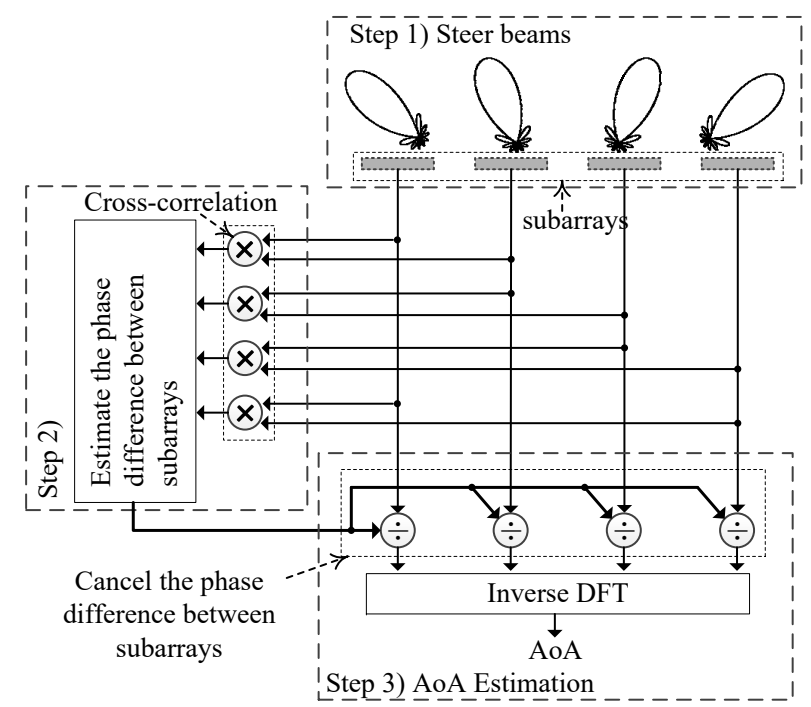

Fig. 4. System diagram of the new unambiguous AoA estimation algorithm developed based on the two new discoveries described in Section III-A. 


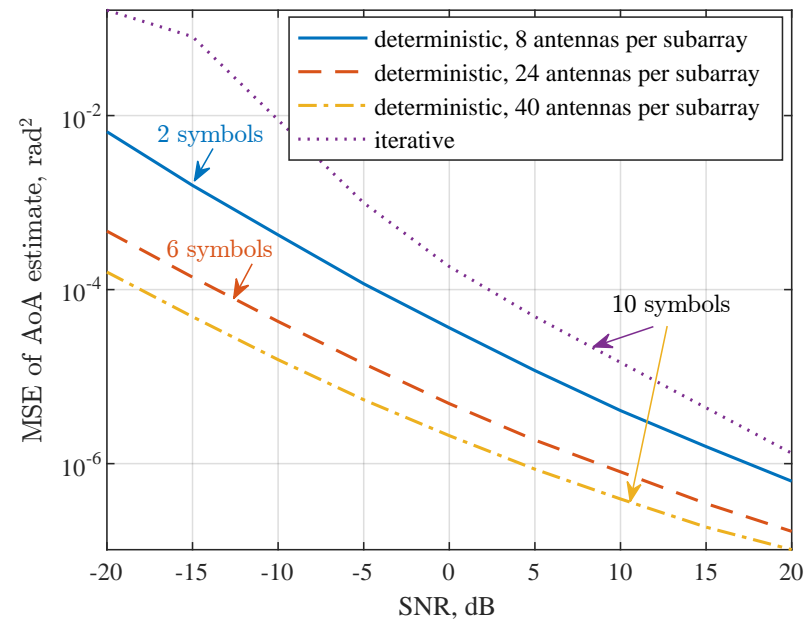

Fig. 5. MSE of AoA estimation vs the received SNR at antennas, where the new AoA estimation approach in Section III; referred to as "deterministic", is compared with the state-of-the-art approach, referred to as "iterative" [9]. 


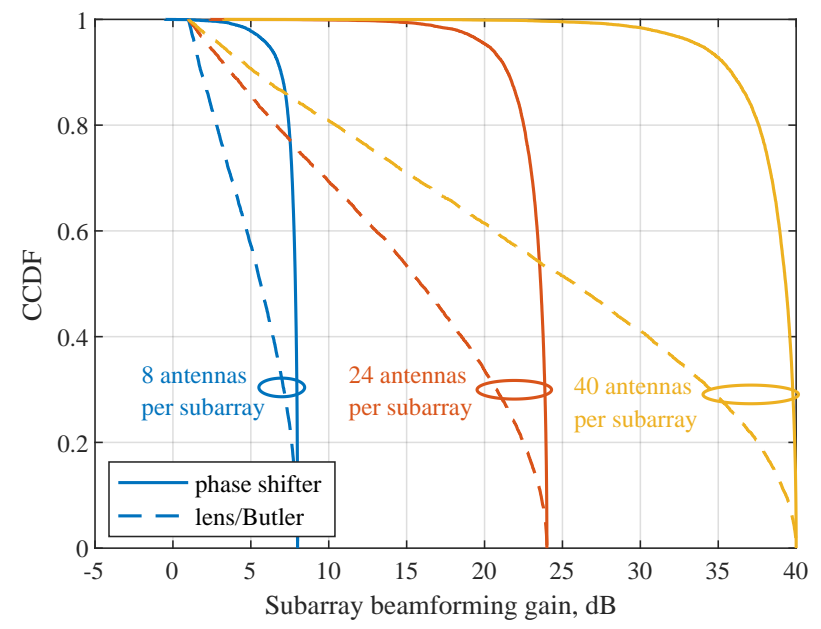

Fig. 6. CCDF of subarray gain based on the AoA estimates in Fig. 5. 